\title{
Resultados maternos y perinatales del manejo conservador de la rotura prematura de membranas en gestantes de 24 a 33 semanas
}

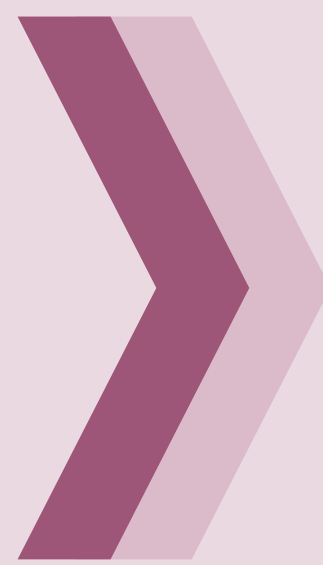

\section{Maternal and perinatal outcomes of conservative management of premature rupture of membranes in pregnancies of 24-33 weeks}

\section{Alan Francis Miranda-Flores ${ }^{1}$}

1. Médico ginecólogo obstetra, asistente del servicio de Obstetricia del Instituto Nacional Materno Perinatal. Lima, Perú.

\section{RESUMEN}

INTRODUCción. El parto prematuro es la causa principal de morbilidad y mortalidad neonatales. Овлетіvo. Determinar los resultados maternos y perinatales del manejo conservador de la ruptura prematura de membranas pretérmino en gestantes de 24 a 33 semanas, en el período 2010-2011, en el Instituto Nacional Materno Perinatal (INMP). Material Y Método. Estudio no experimental, observacional, retrospectivo, transversal, descriptivo desarrollado en el INMP. La población estuvo compuesta por gestantes pretérmino con ruptura prematura de membranas. Se recolectó datos registrados en las historias clínicas. Resultados. Fueron 142 casos los que culminaron el parto por cesárea (71,8\%), con edad de 26,36 $\pm 7,418$ años. Las nuliparas fueron más frecuentes (45,8\%). La edad gestacional promedio fue $29,06 \pm 2,590$ semanas; el control prenatal promedio, 2,35 $\pm 2,101$; el período de latencia promedio, 10,68 $\pm 12,305$ días. Hubo resultados maternos en $34,5 \%$ (49 casos), donde la coriamnionitis fue el más frecuente (23,9\%). Hubo resultados perinatales en $32,4 \%$ (46 casos), donde el sindrome de dificultad respiratoria fue el más frecuente $(21,8 \%)$. El peso promedio al nacer fue de $1653,14 \mathrm{~g} \pm 460,219 \mathrm{~g}$. La mayoría de los recién nacidos pretérminos al nacer tuvieron buen Apgar. El promedio de días de internamiento de la madre fue de 15,30 días $\pm 12,912$. Conclusiones. La coriamnionitis y el síndrome de dificultad respiratoria fueron los resultados maternos $y$ perinatales más frecuentes.

Palabras clave. Resultados maternos, resultados perinatales, ruptura prematura de membranas pretérmino, manejo conservador.

\section{SUMMARY}

InTRODUCtion. Preterm birth is the leading cause of neonatal morbidity and mortality. ОвJеCтIVEs. Determine maternal and perinatal outcomes of conservative management of preterm premature rupture of membranes in pregnancies of 24-33 weeks in the period 2010-2011. Material and method. Non-experimental, observational, retrospective, cross-sectional, descriptive from National Institute Maternal Perinatal. Pregnancies with preterm premature rupture of membranes. I collect it of data from medical records. Results. There were 142 cases, the culmination of the delivery via was the cesarean $(71,8 \%)$, the mean age was $26,36 \pm 7,418$ years. The nulliparous were more frequent $45,8 \%$. The mean gestational age was $29,06 \pm 2,590$ weeks. The mean birth control was $2,35+2,101$. The mean latency period was $10,68 \pm 12,305$ days. Maternal outcomes were 34,5\% (49 cases) where the chorioamnionitis was most frequent $23,9 \%$. Perinatal outcome was $32,4 \%$ (46 cases) where respiratory distress syndrome was the most frequent $21,8 \%$. The mean birth weight was $1653,14 \mathrm{~g} \pm 460,219$. Most preterm infants had good Apgar scores at birth. The mean days of hospitalization of the mother were of 15,30 days $\pm 12,912$. Conclusions. The chorioamnionitis and respiratory distress syndrome were the maternal and perinatal outcomes more frequent.

KeY WORDs. Maternal outcomes, perinatal outcomes, preterm premature rupture of membranes, conservative management. 


\section{INTRODUCCIÓN}

La rotura prematura de membranas (RPM) es la rotura espontánea de las membranas corioamnióticas producidas antes del inicio del trabajo de parto, en una gestación posterior a las 22 semanas de gestación..$^{1-5}$ Se presenta con una frecuencia entre $4 \%$ y $18 \%$ de los partos y es causa de $50 \%$ de los partos pretérminos. ${ }^{6}$ La RPM pretérmino (RPMPT) se define como la rotura antes de las 37 semanas de gestación, representa 3\% de los embarazos y es responsable de una tercera parte de los nacimientos pretérmino. El parto prematuro es la causa principal de morbilidad y mortalidad neonatales. ${ }^{3}$

El pronóstico perinatal y el manejo está relacionado con la edad gestacional al momento en que se produce la RPM. Según el Colegio Americano de Obstetras y Ginecólogos (ACOG, del inglés American College of Obstetricians and Gynecologists), la morbilidad materna principalmente se relaciona con la infección intraamniótica (13\%-60\%) y la endometritis posparto (2 \%-13 \%); en cambio, la morbilidad fetal en pretérminos se relaciona con la enterocolitis necrosante, la sepsis neonatal, el síndrome de dificultad respiratoria y la hemorragia intraventricular. $^{5}$

El manejo conservador de la RPMPT va a depender de la edad gestacional. Tiene mejor beneficio en los casos menores de 32 semanas de edad gestacional, ya que el nacimiento inmediato de recién nacidos con un peso menor de $1500 \mathrm{~g}$ se asocia con elevadas complicaciones relacionadas con la prematuridad. En las últimas décadas, el problema de prematuridad asociado a RPM se ha ido incrementando. Sin embargo, existe todavía controversia acerca del manejo de las gestantes entre 28 y 33 semanas con RPM. Muchos consideran el manejo conservador y la intervención rápida a las 34 semanas, con la finalidad de prolongar el embarazo, para reducir la morbilidad secundaria a la prematuridad, con vigilancia estrecha de la presencia de infección, desprendimiento prematuro de placenta, trabajo de parto y compromiso fetal debido a compresión del cordón umbilical, entre otros.
En cambio, después de las 32 semanas de gestación, el principal riesgo para el neonato es una infección y no las complicaciones de la prematuridad. En general, después de las 32 semanas de gestación, la supervivencia es alta y las secuelas poco comunes. No es recomendable el manejo conservador en gestaciones mayores de 34 semanas. ${ }^{2,4,7-9}$

El manejo conservador se basa en la hospitalización de la paciente, reposo en cama, tactos vaginales restringidos, control de los signos vitales maternos, controles obstétricos, uso de antibióticos profilácticos hasta completar los 7 días, maduración pulmonar -mediante la administración de corticoides; betametasona, $12 \mathrm{mg}$, intramuscular, cada 24 horas, por dos dosis, o dexametasona, $6 \mathrm{mg}$, intramuscular, cada 12 horas, por cuatro dosis- $y$, si es necesario, el uso de tocólisis. 6,9

Sirjana, ${ }^{10}$ en un estudio retrospectivo de 187 recién nacidos prematuros con antecedente de RPM, comparó los resultados entre los recién nacidos pretérminos con RPM y los recién nacidos pretérminos sin historia de RPM y concluyó que la asfixia al nacer, el síndrome de dificultad respiratoria, el apnea del sueño y la neumonía son comunes en ambos grupos pero no estadísticamente significativo $(p>0,05)$ en el grupo de casos. Gómez ${ }^{11}$ realizó un estudio descriptivo prospectivo en mujeres con edad gestacional entre 24 y 33 semanas con diagnóstico de RPMPT, que incluyó 35 gestantes en un período de 12 meses, y encontró coriamnionitis (12 casos; $34,3 \%$ ), sepsis neonatal $(6 ; 17,1 \%)$, neumonía (3; $8,6 \%)$, hemorragia intraventricular $(3 ; 8,6 \%$ ) y muerte perinatal (1; 2,9\%).

En la actualidad, el nacimiento de un pretérmino en Perú se está convirtiendo en un problema de salud pública. Por tanto, el motivo de este estudio es conocer los resultados materno-perinatales que se están dando en estos pacientes para tener un mayor conocimiento de ellos, ya que el Instituto Nacional Materno Perinatal (INMP) es un hospital de referencia de alta complejidad y altamente especializado, que recibe la gran mayoría de los pacientes con RPMPT y, a su vez, tiene un servicio de Obstetricia donde van estos pacientes para su estudio y evaluación. 


\section{MÉTODOS}

Se realizó un estudio no experimental, observacional, retrospectivo, transversal y descriptivo. La población de estudio fueron las gestantes de 24 a 33 semanas con RPMPT que recibieron manejo conservador y fueron atendidos en el INMP en el período 2010-2011.

$\triangle$ Criterios de inclusión: gestantes pretérmino de 24 a 33 semanas, RPMPT, feto únicos, historia clínica disponible y con información requerida completa, que recibieron manejo conservador para la RPMPT, y pacientes atendidas en el INMP.

$\Delta$ Criterios de exclusión: diagnóstico prenatal de malformaciones fetales, embarazos pretérminos menores de 24 semanas y de 34 semanas o más, historia clínica no disponible y/o con información requerida incompleta, embarazos gemelares, óbitos fetales $y$ referencia de la paciente.

Las variables del estudio tomados fueron las siguientes: manejo conservador de la RPMPT, resultados maternos (corioamnionitis, endometritis puerperal, infección de sitio operatorio, sepsis materna, muerte materna, desprendimiento prematuro de placenta normoinserta y hemorragia postparto), resultados perinatales (sepsis neonatal, síndrome de dificultad respiratoria, muerte perinatal, asfixia perinatal, hemorragia intraventricular, enterocolitis necrosante y neumonía), edad materna, edad gestacional, paridad, período de latencia, parto vaginal, cesárea, control prenatal, peso al nacer, días de internamiento y Apgar.

Se realizó la técnica de observación documental en historias clínicas y se utilizó un formulario de registro. Los datos se registraron en una base de datos elaborada en la hoja de cálculo del programa SPSS versión 17.0, se tomó en cuenta todos las variables e indicadores. El análisis descriptivo y analítico se realizó con el apoyo del paquete estadístico; para la presentación de los datos, se utilizó fundamentalmente la frecuencia, y para el análisis de la información, los porcentajes.

\section{RESULTADOS}

La vía de culminación del parto en $71,8 \%$ de los 142 casos fue la cesárea. La edad materna promedio fue 26,36 años, con una edad materna mínima de 14 años y una máxima de 50 años; $45,8 \%$ de los casos fueron nulíparas. La edad gestacional promedio fue 29,06 semanas, con una edad gestacional mínima de 24 semanas y una máxima de 33 semanas. El número de controles prenatales fue 2,35 controles, donde la falta de controles ( 0 controles) fue lo más frecuente $(26,76 \%)$. El período de latencia promedio fue 10,68 días, con un período de latencia mínimo de 2 días y un máximo de 74 días. Tablas 1 y 2.

En relación al peso al nacer, el promedio fue 1653,14 g; el peso mínimo, 646 g y el máximo, 2584 g. En cuanto al puntaje de Apgar en los recién nacidos pretérminos, fue de 7 a 10 , en $65,5 \%$ al minuto y en $90,1 \%$, a los cinco minutos. El número de días de internamiento promedio de

\begin{tabular}{|c|c|c|}
\hline Variables & $\mathrm{N}$ & $\%$ \\
\hline Gestantes con manejo conservador & 142 & 100,0 \\
\hline \multicolumn{3}{|l|}{$\Delta$ Vía de culminación del parto } \\
\hline - Cesárea & 102 & 71,8 \\
\hline - Parto vaginal & 40 & 28,2 \\
\hline \multicolumn{3}{|l|}{$\Delta$ Paridad } \\
\hline - Nulípara & 65 & 45,8 \\
\hline - Primípara & 36 & 25,4 \\
\hline - Multípara & 36 & 25,4 \\
\hline - Gran multípara & 5 & 3,5 \\
\hline \multicolumn{3}{|l|}{$\Delta$ Apgar al minuto } \\
\hline$-0-3$ & 13 & 9,2 \\
\hline$-4-6$ & 36 & 25,4 \\
\hline$-7-10$ & 93 & 65,5 \\
\hline \multicolumn{3}{|l|}{$\Delta$ Apgar a los 5 minutos } \\
\hline$-0-3$ & 5 & 3,5 \\
\hline$-4-6$ & 9 & 6,3 \\
\hline$-7-10$ & 128 & 90,1 \\
\hline
\end{tabular}


Tabla 2. Características maternoperinatales de la población de estudio

$\begin{array}{lccrr} & \text { Media } & \text { Desviación estándar } & \text { Mínimo } & \text { Máximo } \\ \Delta \text { Edad materna } & 26,36 \text { años } & 7418 & 14 & 50 \\ \Delta \text { Edad gestacional } & 29,06 \text { semanas } & 2590 & 24 & 33 \\ \Delta \text { Control prenatal } & 2,35 & 2101 & 0 & 9 \\ \Delta \text { Período de latencia } & 10,68 \text { días } & 12305 & 2 & 74 \\ \Delta \text { Peso al nacer } & 1653,14 \text { gramos } & 460219 & 646 & 2584 \\ \Delta \text { Días de internamiento } & 15,30 \text { días } & 12912 & 3 & 78\end{array}$

la madre fue 15,30 días, con un rango de 3 a 78 días. Tabla 2.

Los resultados maternos se dieron en 49 casos $(34,5 \%)$. La coriamnionitis fue el más frecuente $(23,9 \%)$, seguida por la infección de sitio operatorio (10,6\%), el desprendimiento prematuro de placenta $(2,8 \%)$, la sepsis materna $(2,1 \%)$, la endometritis puerperal $(2,1 \%)$ y la hemorragia postparto $(1,4 \%)$. Tabla 3.

Los resultados perinatales se dieron en 46 casos $(32,4 \%)$. El síndrome de dificultad respiratoria fue el más frecuente $(21,8 \%)$, seguido por la neumonía neonatal $(14,8 \%)$, la hemorragia intraventricular $(11,3 \%)$, la asfixia neonatal (9,2\%), la muerte neonatal $(4,9 \%)$, la sepsis neonatal $(3,5 \%)$ y la enterocolitis necrosante (0,7\%). Tabla 4.

\begin{tabular}{|c|c|c|}
\hline Resultados maternos & $\mathrm{N}$ & $\%$ \\
\hline$\triangle$ Corioamnionitis & 34 & 23,9 \\
\hline$\Delta$ Infección del sitio operatorio & 15 & 10,6 \\
\hline$\Delta$ Desprendimiento prematuro de placenta & 4 & 2,8 \\
\hline$\triangle$ Sepsis materna & 3 & 2,1 \\
\hline$\Delta$ Endometritis puerperal & 3 & 2,1 \\
\hline$\Delta$ Hemorragia posparto & 2 & 1,4 \\
\hline$\Delta$ Muerte materna & 0 & 0,0 \\
\hline
\end{tabular}

\section{DISCUSIÓN}

En la actualidad, en el INMP, existe un protocolo del manejo conservador de la RPM entre las 24 y 33 semanas. La RPM es una de las causas más importantes del parto pretérmino y este, a su vez, de la morbimortalidad neonatal. En el presente estudio se ha encontrado 142 casos de gestantes con RPMPT entre las 24 y 33 semanas que recibieron tratamiento conservador, a diferencia de otros estudios con número de casos menor, como el de Gómez ${ }^{11}$ ( 35 casos), de Ovalle ${ }^{12}$ (135 casos), de $\mathrm{Cruz}^{13}$ ( 46 casos), de Stewart ${ }^{14}$ (78 casos), de Saavedra ${ }^{15}$ (52 casos) o de $\operatorname{Barco}^{16}$ (52 casos), u otros estudios con mayor número, como el de Sirjana ${ }^{10}$ (187 casos) y Melamed ${ }^{17}$ (488 casos).

La vía de culminación de la gestación fue cesárea en 102 casos $(71,8 \%$ y parto vaginal en 40 casos
Tabla 4. Resultados perinatales de las gestantes con ruptura prematura de membranas pretérmino con manejo conservador

$\begin{array}{lrr}\text { Resultados perinatales } & \text { N } & \% \\ \Delta \text { Síndrome de dificultad respiratoria } & 31 & 21,8 \\ \Delta \text { Neumonía neonatal } & 21 & 14,8 \\ \Delta \text { Hemorragia intraventricular } & 16 & 11,3 \\ \Delta \text { Asfixia neonatal } & 13 & 9,2 \\ \Delta \text { Muerte neonatal } & 7 & 4,9 \\ \Delta \text { Sepsis neonatal } & 5 & 3,5 \\ \Delta \text { Enterocolitis necrosante } & 1 & 0,7\end{array}$


$(28,2 \%)$. Son pocos los estudios que indican el tipo de parto en estas pacientes; en el de Ovalle, ${ }^{12}$ el parto vaginal fue más frecuente (50,1\%); en el de Barco, ${ }^{16}$ el parto vaginal (56\%); en el de Melamed, ${ }^{17}$ la cesárea; en el de Riveros, ${ }^{18}$ el parto vaginal (54\%). Según el protocolo del INMP, en las gestaciones de 26 a 31 semanas + 6 días está indicada la cesárea y en las gestaciones menores de 26 y mayores de 31 está indicado el parto vaginal, pero como va a depender de las condiciones de la madre como la del feto, por lo cual la cesárea representa un mayor porcentaje.

En relación a las características de la población de estudio, la edad materna promedio fue 26,36 años $\pm 7,418$; el mayor porcentaje se encuentra entra las edades 26 a 31 años con $27,5 \%$, está acorde con la edad promedio encontrada en los estudios revisados por Sirjana et al.,10 Ovalle et al.,12 Cruz et al., ${ }^{13}$ Melamed, ${ }^{17}$ Morgan ${ }^{19}$ y Furman. ${ }^{20}$

En cuanto a la paridad, la mayoría de casos ocurrieron en gestantes nulíparas (65 casos; $45,8 \%$ ), a diferencia de la mayoría de trabajos de investigación, que fueron en multíparas.

La edad gestacional promedio en el estudio fue de 29,06 semanas $\pm 2,59$ semanas, donde la mayor frecuencia correspondió a las 30 semanas (16,20\%); la que está dentro de los rangos encontrados en los trabajos de revisión de Gómez ${ }^{11}$ (28-31 semanas), Ovalle $^{12}$ (30,6 semanas), Cruz ${ }^{13}$ (31,4 semanas) y $\operatorname{Barco}^{16}$ (29-32 semanas).

En cuanto a los controles prenatales que se realizaron las gestantes, el promedio fue de 2,35 \pm 2,101 controles. El número de controles realizado más frecuente fue $0(26,76 \%)$, seguido de 2 $(23,24 \%)$.

En el estudio el período de latencia promedio fue 10,68 días $\pm 12,305$, con período de latencia mínimo de 2 días y uno máximo de 74 días; valor superior a lo descrito en otros trabajos como los de Gómez ${ }^{11}$ (7 días), Ovalle ${ }^{12}$ (8,6 días), Barco ${ }^{16}$ (7 días) y Melamed ${ }^{17}$ (8,2 días).

Los resultados maternos se presentaron en $34,5 \%$ (49 casos) de las gestantes del presente estudio. La complicación más frecuente fue la coriamnionitis (34 casos; 23,9\%), seguida de la infección del sitio operatorio $(15 ; 10,6 \%)$ y el desprendimiento prematuro de placenta $(4 ; 2,8 \%)$. La coriamnionitis también fue el resultado materno más frecuente en otros estudios como los de Gómez ${ }^{11}(34,3 \%)$, $\operatorname{Cruz}^{13}(25,9 \%)$, Barco (28,8\%), Riveros ${ }^{18}(25 \%)$ y Furman $^{20}(16,2 \%)$.

Los resultados perinatales se presentaron en $32,4 \%$ (46 casos) de los recién nacidos pretérminos del presente estudio. La complicación más frecuente fue el sindrome de dificultad respiratoria (31 casos; $21,8 \%$ ), seguida de la neumonía neonatal (21; $14,8 \%$ ), la hemorragia intraventricular (16; $11,3 \%$ ) y la asfixia neonatal (13; 9,2 \%). El síndrome de dificultad respiratoria también fue el resultado perinatal más frecuente en la mayoría de los trabajos revisados, como los de Sirjana, ${ }^{10}$ Cruz, ${ }^{13}$ $\operatorname{Barco}^{16}(25 \%)$, Riveros $^{18}$ (88 \%) y Ariadna ${ }^{21}(70 \%)$.

El peso al nacer promedio fue $1653,14 \mathrm{~g} \pm 460,219$ $\mathrm{g}$, con un peso mínimo de $646 \mathrm{~g}$ y uno máximo de 2584 g, en comparación con los estudios revisados que fueron mayores. En el estudio de Sirjana,10 el peso al nacer promedio fue $2160 \mathrm{~g}$; en el de Ovalle, 121903 en nacidos por parto vaginal y 1823 en nacidos por cesárea g; en el de Barco, ${ }^{16} 1834 \mathrm{~g}$; en el de Riveros, ${ }^{18} 43 \%$ de 1500 a $2000 \mathrm{~g}$ (43\%); Furman, ${ }^{20} 34 \%$ de 2001 a $2500 \mathrm{~g}$.

Los recién nacidos pretérminos en el presente estudio presentaron un puntaje Apgar bueno. En el Apgar al minuto, 65,5\% de los recién nacidos pretérminos tuvieron un puntaje de $7-10$ y $25,4 \%$, puntaje de 4-6; en el Apgar a los 5 minutos, 90,1\% tuvieron un puntaje de 7-10 y 6,3\%, puntaje de 4-6.

En relación a los días de internamiento el promedio fue de 15,30 días $\pm 12,912$ días, donde el mínimo de días de internamiento es de 3 días y el máximo fue de 78 días, no hay estudios que refieren sobre este punto.

En conclusión, en el manejo conservador de las gestantes con rotura prematura de membranas pretérmino entre las 24 y las 33 semanas en el INMP, los resultados materno y perinatal más frecuentes fue la coriamnionitis y el síndrome de dificultad respiratoria, respectivamente; la vía de culminación de parto, con una alta frecuencia, fue la cesárea y la mayoría de los recién nacidos pretérmino tuvieron un buen Apgar. 


\section{REFERENCIAS BIBLIOGRÁFICAS}

I. Doren A, Carvajal J. Alternativas de manejo expectante de la rotura prematura de membranas antes de la viabilidad en embarazos únicos. Rev Chil Obstet Ginecol. 2012;77:225-234.

2. Waters TP, Mercer B. Preterm PROM: Prediction, prevention, principles. Clin Obstet Gynecol. 20 I I;54(2):307-3I 2.

3. Cifuentes R. Ginecología y Obstetricia basadas en las nuevas evidencias. 2. ${ }^{a}$ edición. Bogotá: Distribuidora Editorial Médica; 2009.

4. Romero-Arauz JF, Álvarez-Jiménez G, Ramos León JC. Manejo de ruptura prematura de membranas pretérmino. Ginecol Obstet Mex. 2009;77(Suppl.7):SI77-209.

5. ACOG Practice Bulletin N. 80 : Premature rupture of membranes. Obstet Gynecol. 2007;109(4):1007-1019.

6. Instituto Nacional Materno Perinatal. Guías de Práctica Clínica y de Procedimientos en Obstetricia y Perinatología. Lima: INMP; 2010.

7. Jain VD, Sciscione AS. Considerations in membrane resealing after preterm PROM. Clin Obstet Gynecol. 201 I;54(2):35I-357.

8. Clark EA, Varner M. Impact of preterm PROM and its complications on long-term infant outcomes. Clin Obstet Gynecol. 201 I;54(2):358-369.

9. Thomas J, Garite M. Management of premature rupture of membranes. Clin Perinatol. 200I;28(4):837-847.

10. Sirjana K, Zhang W, Rajbhandari N, Dahal D. A Comparative study of outcome of preterm neonate with and without history of preterm premature rupture of membrane. Nepal Med Coll J. 2009; I I (2):99-103.

11. Gómez Dávila JG, Barco Burgos MC, Úsuga Berrío YA, Araque Fernández AA. Resultados perinatales de las mujeres con ruptura prematura de membranas ovulares entre las semanas 24 y 34 de la gestación sometidas a manejo expectante en el Hospital Universitario San Vicente de Paúl, Medellín, Colombia. latreia. 2008;2I(4-S):SI8.

12. Ovalle A, Martínez MA, Fuentes A, Kakarleka E, Aspillaga C, Saavedra R, Medel S. Resultado neonatal adverso en la rotura prematura de membranas pretérmino según el modo del parto. Rev Chil Obstet Ginecol. 2007;72(3):144-I53.

13. Cruz LG, Uribasterra CA, Ramírez FN, González BA. Rotura prematura de membranas pretérmino. Estudio comparativo 2002-2003. Hospital DocenteVladimir I. Lenin. Correo Científico Médico de Holguín. 2006; I0(I).

14. Stewart CJ, Tregoning K, Moller G, Wainwright H. Preterm prelabour rupture of the membranes before 28 weeks: better than feared outcome of expectant management in Africa. Eur J Obstet Gynecol Reprod Biol. 2006; I26(2):186-189.

15. Saavedra D, Valdes S, Essien J, De la Torre Y. Morbimortalidad perinatal de la rotura prematura de membrana en el embarazo pretérmino. Rev Clin Investig Ginecol Obstet. 2006;33(3):102106.

16. Barco MC, Noreña EA, Ocampo OJ, Salcedo ER. Manejo conservador de la ruptura prematura de membranas pretérmino. Rev Colomb Obstet Ginecol.1999;50:157-162.

17. Melamed N, Ben-Haroush A, Pardo J, Chen R, Hadar E, Hod M, YogevY. Expectant management of preterm premature rupture of membranes: is it all about gestational age? Am J Obstet Gynecol. 20II;204(I):48.el-8.

18. Riveros J. Resultados perinatales en la rotura prematura de membranas. Rev Nac (Itauguá). 20I I;3(I):35-4I.

19. Morgan O, Gómez S, Valenzuela G, Gonzales B, Quevedo C, Osuna R. Factores sociodemográficos y obstétricos asociados con rotura prematura de membranas. Ginecol Obstet Mex. 2008;76(8):468-475.

20. Furman B, Shoham I, Bashiria A, Ereza O, Mazora M. Significación clínica y resultado de la rotura prematura de membranas pretérmino: estudio basado en la población. Eur J Obstet Gynecol Reprod Biol. 2001;1:122-29.

21. Hernández y Ballinas A, López Farfán JA, Gámez Guevara C. Comparación de resultados maternos y perinatales en el tratamiento conservador de la rotura prematura de membranas pretérmino entre el uso de eritromicina y clindamicina. Ginecol Obstet Mex. 201 I;79(7):403-4I0.

22. Vidaeff C, Ramin SM. Antenatal corticosteroids after preterm premature rupture of membranes. Clin Obstet Gynecol. 201 I; 54(2):337-343.

23. Singh K, Mercer B. Antibiotics after preterm premature rupture of membranes. Clin Obstet Gynecol. 201 1;54(2):344-350.

24. Manuck TA, Maclean CC, Silver RM, Varner MW. Preterm premature rupture of membranes: does the duration of latency influence perinatal outcomes? Am J Obstet Gynecol. 2009; 20I(4):4I4.el-e6.

25. Gabbe SG, Niebyl JR, Simpson JL. Obstetrics. Normal and problem pregnancies.4th edition. Madrid: Marbán Libros S.L.; 2006.

26. Kenyon S, Boulvain M, Neilson JP. Antibióticos para la rotura prematura de membranas. Cochrane Database of Systematic Reviews. 2013 Issue 12. Art. N.:: CD00 1058. DOI: 10.1002/I465/858.CD00I058.

27. Cunningham FG, Leveno KJ, Bloom SL, Hauth JC, Gilstrap LC, Wenstrom KD. Obstetricia de Williams. 22. ${ }^{a}$ edición. México D.F.: McGraw-Hill Interamericana; 2006.

28. Hnat MD, Mercer BM, Thurnau G, Goldenberg R, Thom EA, Meis PJ, Moawad AH, lams JD, Van Dorsten JP. Perinatal outcomes in women with preterm rupture of membranes between 24 and 32 weeks of gestation and a history of vaginal bleeding. Am J Obstetrics and Gynecology. 2005; 193:164-168.

\section{Correspondencia}

Dr.Alan Francis Miranda-Flores

alanmiranda04@hotmail.com

\section{Conflictos de interés}

Los autores declaran no tener conflictos de interés durante el planteamiento, ejecución de la investigación y la elaboración del artículo para su publicación.

Fecha de recepción: 14 de abril de 2014

Fecha de aceptación: 19 de mayo de 2014 